\title{
Viscosity and the equation of state in high energy heavy-ion reactions
}

\author{
W. Schmidt, ${ }^{*}$ U. Katscher, B. Waldhauser, J. A. Maruhn, H. Stöcker, and W. Greiner \\ Institut für Theoretische Physik, J. W. Goethe-Universität, Robert-Mayer-Str. 8-10, D-6000 Frankfurt-am-Main, Germany
}

(Received 5 March 1992)

\begin{abstract}
Viscous hydrodynamic calculations of high energy heavy-ion collisions ( $\mathrm{Nb}-\mathrm{Nb}$ and $\mathrm{Au}-\mathrm{Au}$ ) from 200 to $800 \mathrm{MeV} /$ nucleon are presented. The resulting baryon rapidity distributions, the in-plane transverse momentum transfer (bounce-off), and the azimuthal dependence of the midrapidity particles (off-plane squeeze out) compare well with Plastic Ball data. We find that the considered observables are sensitive both to the nuclear equation of state and to the nuclear shear viscosity $\eta$. Transverse momentum distributions indicate a high shear viscosity $\left(\eta \approx 60 \mathrm{MeV} / \mathrm{fm}^{2} c\right)$ in the compression zone, in agreement with nuclear matter estimates. The bulk viscosity $\zeta$ influences only the entropy production during the expansion stage; collective observables like flow and $d N / d Y$ do not depend strongly on $\zeta$. The recently observed off-plane $\left(\phi=90^{\circ}\right)$ squeeze-out, which is found in the triple-differential rapidity distribution, exhibits the strongest sensitivity to the nuclear equation of state. It is demonstrated that for very central collisions, $b=1 \mathrm{fm}$, the squeeze-out is visible even in the double-differential cross section. This is experimentally accessible by studying azimuthally symmetric events, as confirmed recently by data of the European $4 \pi$ detector collaboration at Gesellchaft für Schwerionforschung Darmstadt.
\end{abstract}

PACS number(s): 25.75. $+\mathrm{r}$

\section{INTRODUCTION}

The key mechanism for creating hot, dense nuclear matter in the laboratory is the formation of nuclear compression waves and the study of the resulting collective flow in relativistic heavy-ion collisions (for a survey see Ref. [1]). Nuclear fluid dynamics had been the first theory to predict that such novel states of nuclear matter are formed in nuclear collisions [2,3]. The collective flow, predicted as a consequence of the buildup of high pressure in the dense matter [4,5], has indeed been discovered in a series of pioneering experiments at LBL's Bevalac, using the Plastic Ball and Streamer Chamber spectrometers: The bounce-off effect [4] and the resulting in-plane flow were first observed $[6,7]$. The squeeze-out of the hot participant matter perpendicular to the reaction plane ("off-plane") [4,5] has only recently been discovered experimentally [8].

These experiments are now being succeeded by a new generation of apparatus installed at the recently completed SIS facility at GSI. In particular, a high-granularity $4 \pi$ detector system with appropriate azimuthal and polar resolution has been constructed by a large collaboration of scientists from many different European countries, which has focused on the measurement of tripledifferential cross sections with high statistics. They have devised a new method to isolate the most central reactions, with impact parameters $b \leq 1 \mathrm{fm}$, which relies on the measurement of exceptionally good azimuthal sym-

*Former address: Gesellschaft für Schwerionenforschung, D-6100 Darmstadt 11. Present address: Hoechst AG, Abt. EMR, D-6230 Frankfurt am Main 80. metry. Both these data, as well as spectrometer data by Claesson et al. [9] with very hard centrality triggers, seem to allow for a direct observation of the longstanding hydrodynamic prediction [1-5] of the hitherto unobserved "pancake" or "doughnut" shape events in very central collisions.

The potential for probing fundamental properties of nuclear matter far from the ground state, namely, the nuclear viscosity and the equation of state, has stimulated earlier investigations $[10,11]$ and also the present investigation of heavy-ion collisions in viscous nuclear fluid dynamics. Here we emphasize that a simultaneous investigation of all the distinct observed flow effects in one selfconsistent approach is performed.

\section{VISCOUS NUCLEAR FLUID DYNAMICS}

The equations of motion for the viscous, nonrelativistic nuclear fluid can be written as a system of five continuity equations,

$$
\begin{aligned}
& \frac{\partial \rho}{\partial t}+\frac{\partial}{\partial x_{i}}\left(\rho v_{i}\right)=0, \\
& \frac{\partial\left(\rho v_{i}\right)}{\partial t}+\frac{\partial}{\partial x_{j}}\left(\rho v_{i} v_{j}\right)=\frac{\partial}{\partial x_{j}} \sigma_{i j}-\rho \frac{\partial \Phi}{\partial x_{i}}, \\
& \frac{\partial \epsilon}{\partial t}+\frac{\partial}{\partial x_{i}}\left(\epsilon v_{i}\right)=\frac{\partial}{\partial x_{j}}\left(v_{i} \sigma_{i j}\right)-\frac{\partial q_{i}}{\partial x_{i}}-\rho v_{i} \frac{\partial \Phi}{\partial x_{i}},
\end{aligned}
$$

where $\rho, \rho v_{i}$, and $\epsilon=\rho\left[v^{2} /(2 m)+E(\rho, T)\right]$ are the local densities for baryon number, momentum, and energy, respectively. $v_{i}$ is the local velocity, $m$ the nucleon mass, $E(\rho, T)$ is the internal energy, and $q_{i}=-\kappa \partial T / \partial x_{i}$ is the vector of heat transport according to Fourier's law, where $\kappa$ is the coefficient of thermal conduction. The Yu- 
kawa and Coulomb potentials are denoted by $\Phi$.

Using a Newtonian ansatz, the stress tensor $\sigma_{i j}$ can be written as

$\sigma_{i j}=-p \delta_{i j}+\eta\left(\frac{\partial v_{i}}{\partial x_{j}}+\frac{\partial v_{j}}{\partial x_{i}}-\frac{2}{3} \delta_{i j} \frac{\partial v_{k}}{\partial x_{k}}\right)+\zeta \delta_{i j} \frac{\partial v_{k}}{\partial x_{k}}$,

with $\eta$ and $\zeta$ being the coefficients of shear and bulk viscosity. These are, in general, functions of density and temperature [12]. In our model, however, they are set constant $[11,13]$.

The pressure $p$ is calculated by

$$
p=p(\rho, T)=\left.\rho^{2} \frac{\partial E(\rho, T)}{\partial \rho}\right|_{s}
$$

from the energy per particle $E(\rho, T)$ (here loosely referred to as the equation of state). $E(\rho, T)$ is commonly split into two parts, the compressional and the thermal excitation energy:

$$
E(\rho, T)=E_{c}(\rho)+E^{*}(\rho, T) .
$$

Only the thermal energy of a free nonrelativistic Fermi gas has been included in $E^{*}(\rho T)$ :

$$
E^{*}(\rho, T)=E_{\mathrm{th}}(\rho, T)=E_{F}(\rho, T)-E_{F}(\rho, T=0)
$$

with

$$
E_{F}(\rho, T)=\frac{g}{\rho \hbar^{3}}\left(\frac{m}{2}\right)^{1 / 2} \int_{0}^{\infty} \frac{\sqrt{\epsilon} d \epsilon}{e^{(\epsilon-\mu) / T}+1},
$$

where $\mu$ is the nucleon chemical potential and $g$ is the spin-isospin degeneracy factor ( $g=4$ for nucleons).

We use the standard quadratic ansatz [2] for the compressional part of the equation of state:

$$
E_{c}(\rho)=\frac{K}{18 \rho_{0}^{2}}\left(\rho-\rho_{0}\right)^{2}+w_{0}
$$

Compressional part of the EOS:

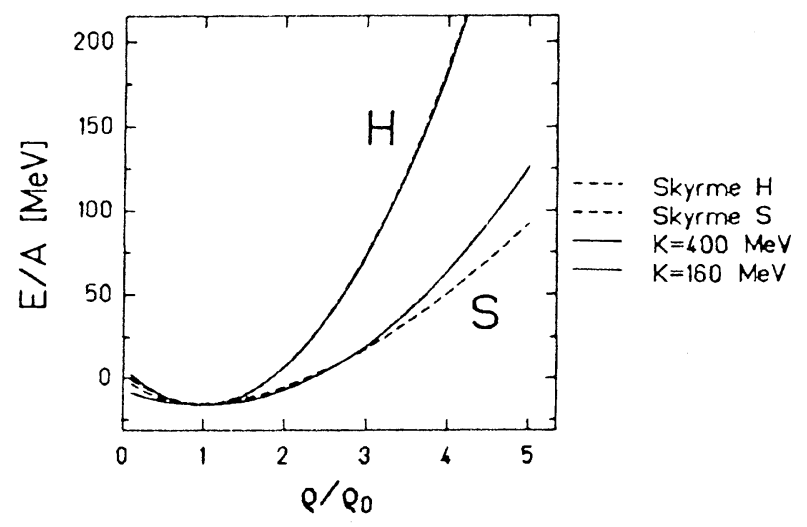

FIG. 1. Sketch of the compressional energy $E_{c}(\rho)$, showing two different parametrizations of the equation of state as used in QMD and nuclear fluid-dynamics calculations, both of them with a hard and a soft variant.
TABLE I. Input parameters for theoretical models. The free nucleon-nucleon cross section $\sigma_{N N}$ may be substituted in certain calculations by the effective in-medium cross section $\sigma_{\text {eff }} U(\rho)$ is the mean-field potential, $E(\rho, T)$ is the equation of state.

\begin{tabular}{lll}
\hline \hline \multicolumn{1}{c}{ Model } & $\begin{array}{c}\text { Macroscopic } \\
\text { properties }\end{array}$ & \multicolumn{1}{c}{$\begin{array}{c}\text { Microscopic } \\
\text { properties }\end{array}$} \\
\hline MD & & Classical potential \\
Cascade & & $\sigma_{N N}$ \\
VUU/QMD & $U(\rho)$ & $\sigma_{N N}$ or $\sigma_{\text {eff }}$ \\
Shock & $E(\rho, T)$ & \\
Nuclear fluid & $E(\rho, T)$ & $\eta, \zeta, \kappa$ \\
$\quad$ dynamics & & \\
\hline \hline
\end{tabular}

where $w_{0}$ denotes the equilibrium binding energy of nuclear matter and $\rho_{0}$ is the equilibrium density. We used $w_{0}=-16 \mathrm{MeV} /$ nucleon and $\rho_{0}=0.16 \mathrm{fm}^{-3}$.

$K$ is the incompressibility constant. To allow for comparison of our results with a microscopic model, namely, the Vlasov-Uehling-Uhlenbeck (VUU) theory and quantum molecular dynamics (QMD) [14-16], we use two different values of $K$ : (a) the soft equation of state, where $K=160 \mathrm{MeV}$, and (b), the hard equation of state, where $K=400 \mathrm{MeV}$.

Note that Skyrme interactions have been employed in the VUU, QMD, and time dependent Hartree-Fock calculations. Refer to Fig. 1 for a sketch of both types of equation of state.

In nuclear fluid dynamics, the equation of state covers the equilibrium properties of nuclear matter, whereas transport coefficients (mainly the shear viscosity $\eta$ ) describe dissipative, nonequilibrium (mean-free-path) effects. Similar relationships exist for the parameters of other theoretical models, which are summarized in Table I. The thermal conductivity $\kappa$ is neglected for the time being.

\section{FRAGMENT FORMATION}

Since the basic assumptions of nuclear fluid dynamics (i.e., local thermal equilibrium and short mean free path) are no longer justified at a late stage of the reaction, the hydrodynamic calculation is abandoned, if the average density decreases to about $\rho_{0} / 2$ : The nuclear fluid is assumed to freeze out. The formation of free nucleons and clusters of nucleons is computed in chemical equilibrium, with conservation of baryon number and energy per particle. The equilibrium is established in a reduced volume $V=V_{0}-\sum_{i} n_{i} V_{i}$, where $n_{i}$ is the number of particles of sort $i$ and $V_{i}$ is their volume [17,21].

So far, only six particles are considered in the calculation, namely, $p, n, d, t,{ }^{3} \mathrm{He}$, and ${ }^{4} \mathrm{He}$. It would be very interesting to also consider the excited state of the deuteron, $d^{*}[18]$. However, for the sake of simplicity, $d^{*}$ is neglected in the present paper, but remains an important point for future investigations. The chemical breakup calculation yields particle numbers and temperatures for each fluid cell. To calculate differential cross sections, the thermal momentum distribution for each cell has to 
be Lorentz transformed to the laboratory frame, assuming that the particles in the cell are forming a free gas with isotropic expansion in the local rest frame. Particle interactions and decay of instable particles are neglected.

The resulting invariant triple-differential cross section $(1 / p) \partial^{3} \sigma / \partial E \partial \Omega$ can be used to compute particle spectra as well as various other observables.

\section{OBSERVABLES}

From the baryon density $\rho$, momentum density $\mathbf{M}=\rho \mathbf{v}$, energy density $\epsilon$, and from the invariant cross sections $(1 / p) \partial^{3} \sigma / \partial E \partial \Omega \partial \phi$ for the six particle species $p$, $n, d, t,{ }^{3} \mathrm{He}$, and ${ }^{4} \mathrm{He}$ one can compute observables, although it may actually be very difficult to extract them from experimental data. The observables that will be investigated in this paper are as follows.

(i) The flow angle $\Theta_{F}$ is the angle between the beam axis and the principal axis of the weighted coalescenceinvariant flow tensor [5]:

$$
F_{i j}=\sum_{v} \frac{1}{2 m_{v}} p_{i}(v) p_{j}(v)
$$

(ii) The aspect ratio $R_{13}$ is the ratio between the largest and the smallest principal axis of the flow tensor $F_{i j}$. It characterizes the anisotropy of a momentum distribution in each event separately [5].

(iii) The entropy per baryon, $S / A$, can be computed from the thermodynamical relation

$$
\frac{S}{A}=\frac{1}{T}\left(\frac{5}{3} E_{\mathrm{th}}-\mu\right),
$$

where $E_{\text {th }}$ is the thermal energy and $\mu$ the chemical potential. The experimental determination of the entropy is much more difficult [19]. For large entropies, $S / A \geq 5$, it can be approximated [20] by $S / A=3.95-\ln R_{d p}$, where $R_{d p}$ is the ratio of $d$-like to $p$-like particles,

$$
R_{d p}=\frac{d+\frac{3}{2}\left(t+{ }^{3} \mathrm{He}\right)+3{ }^{4} \mathrm{He}}{p+d+t+2\left({ }^{3} \mathrm{He}+{ }^{4} \mathrm{He}\right)} .
$$

For lower entropies, a full quantum statistical treatment including complex unstable fragments is necessary [21].

(iv) The distribution of longitudinal momenta parallel to the beam axis is commonly plotted as $d N / d Y$, the baryon rapidity distribution. It measures the stopping power of the nuclei: For peripheral collisions, a pronounced peak at projectile rapidity indicates the presence of projectile spectators, which do not contribute to thermalization. For collisions at intermediate impact parameter, the peak is more and more shifted towards c.m. rapidity as the number of stopped nucleons increases until finally, for central collisions, almost no nucleons remain at their initial rapidity. The incident nuclei are completely stopped in the c.m. frame and the $d N / d Y$ distribution shows a broad maximum at c.m. rapidity.

(v) The transverse momentum analysis is one of the most important observables, since it investigates collective momentum transfer in the center-of-mass (c.m.) frame. Danielewicz and Odyniec [22] proposed to analyze the projection of transverse momentum transfer per particle to the reaction plane $p_{x} / A$, which is plotted as a function of the rapidity. The slope of the $s$-shaped curve at c.m. rapidity, $d p_{x} / d Y$, is then extrapolated to projectile rapidity. Since it has the dimension of momentum, it can be considered as a measure of the collective momentum transfer of the participants [23]. This quantity is commonly (but imprecisely) denoted as collective flow.

(vi) The azimuthal angular correlation of the fragments with respect to the reaction plane, $p_{x} /\left|p_{\perp}\right|$, can also be plotted as a function of rapidity. Experimental results of Kampert [23] show a strong correlation for heavy fragments, which means that those are emitted preferentially in the collective direction of motion, as had been anticipated long ago by Baumgardt et al. [3].

(vii) Cross sections and particle spectra are calculated and can be directly compared with data. Recent measurements of proton spectra in central collisions of $\mathbf{L a} \rightarrow \mathbf{L a}$ at $246 \mathrm{MeV} /$ nucleon have shown a strong $90^{\circ}$ enhancement [9], even in the double-differential cross section. This is supported by the first results of the European $4 \pi$ spectrometer group at GSI.

(viii) Only recently, a new analysis of Bevalac data has shown that the baryon rapidity distribution is not azimuthally symmetric. When plotting the angular rapidity distribution $d N / d Y d \phi$, one finds a clear peak at $Y_{\text {c.m. }}$ and $\phi=90^{\circ}$ indicating a strong off-plane squeeze out of hot nuclear matter [19], as predicted by early fluid dynamical calculations [5].

\section{RESULTS AND COMPARISON WITH DATA}

\section{A. Time development in nuclear fluid dynamics calculations}

Figure 2 shows the time development of some typical quantities in the reaction $\mathrm{Au}+\mathrm{Au}$ at $400 \mathrm{MeV} /$ nucleon

$A u+A u$ at $400 \mathrm{MeV} / \mathrm{N}, \mathrm{b}=3 \mathrm{fm}$, hard EOS

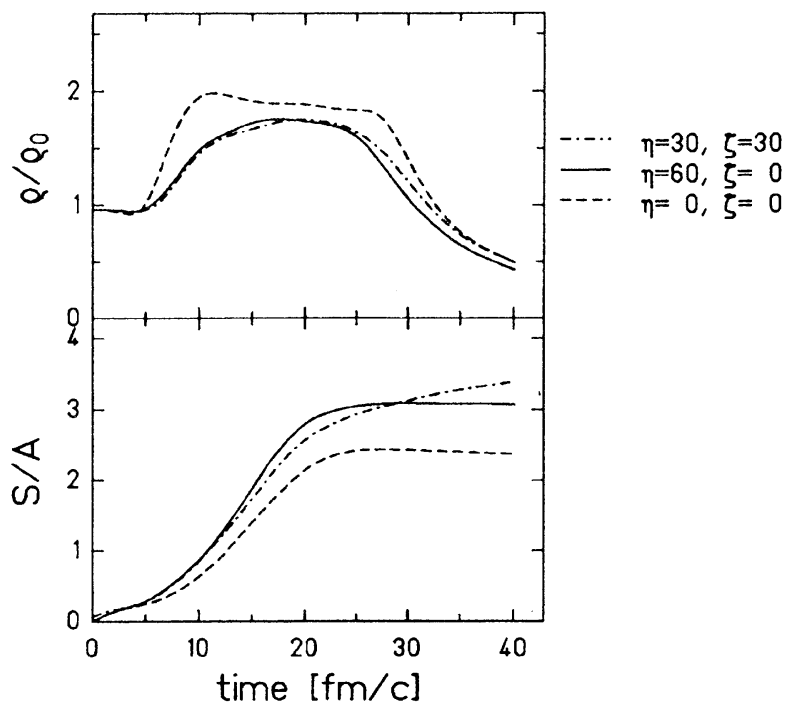

FIG. 2. Compression (top) and entropy per baryon (bottom) in a $\mathrm{Au}+\mathrm{Au}$ reaction at $400 \mathrm{MeV} /$ nucleon, impact parameter $b=3 \mathrm{fm}$, hard equation of state. Dotted: $\eta=0, \xi=0$; straight: $\eta=60, \zeta=0$; dashed: $\eta=30, \xi=30 \mathrm{MeV} / \mathrm{fm}^{2} c$. 
with an impact parameter of $b=3 \mathrm{fm}$, using a hard equation of state. The dashed curve shows data for a nonviscous calculation with $\eta=0$ and $\xi=0$. The solid curve was calculated with a high shear viscosity $(\eta=60$ $\left.\mathrm{MeV} / \mathrm{fm}^{2} c\right)$, but no bulk viscosity $(\xi=0)$. The dasheddotted curve was obtained for $\eta=30$ and $\xi=30$ $\mathrm{MeV} / \mathrm{fm}^{2} c$.

The upper picture [Fig. 2(a)] shows the maximum compression, i.e., the density of the most highly compressed cell in the numerical grid at any given time. Shortly after the collision, the compression starts to rise and reaches a maximum of about $1.9 \rho_{0}$ for nonviscous and $1.6 \rho_{0}$ for viscous calculations: The viscosity tends to decrease the compression. Note the small absolute value of $\rho$, considerably smaller than anticipated from a simple overlay of the densities. This is due both to the repulsive equation of state and to the viscous effects. This maximum is maintained for a certain time, as long as the shock wave runs through the nuclei. When the expansion starts, the compression decreases rapidly. After a certain time, no compressed cells are left. This is the appropriate stage for chemical breakup. In a 400 $\mathrm{MeV} /$ nucleon reaction, it is reached after about $40 \mathrm{fm} / c$.

The lower picture [Fig. 2(b)] shows the average entropy per baryon. Note that most of the entropy is produced during the compression stage. The expansion is adiabatic, unless there is a finite bulk viscosity $\zeta$. Therefore, if $\xi \neq 0$, the entropy per baryon depends on the chemical breakup time.

As one can see from Fig. 3, the influence of the equation of state on these results is low. Here we compare calculations of $\mathrm{Au}+\mathrm{Au}$ at $400 \mathrm{MeV} /$ nucleon with $\eta=0$, $\xi=0$ [Fig. 3(a)] and $\eta=60, \zeta=0 \mathrm{MeV} / \mathrm{fm}^{2} c$ [Fig. 3(b)] for both the hard and the soft equation of state.

$\mathrm{Au}+\mathrm{Au}$ at $400 \mathrm{MeV} / \mathrm{N}, \mathrm{b}=3 \mathrm{fm}$

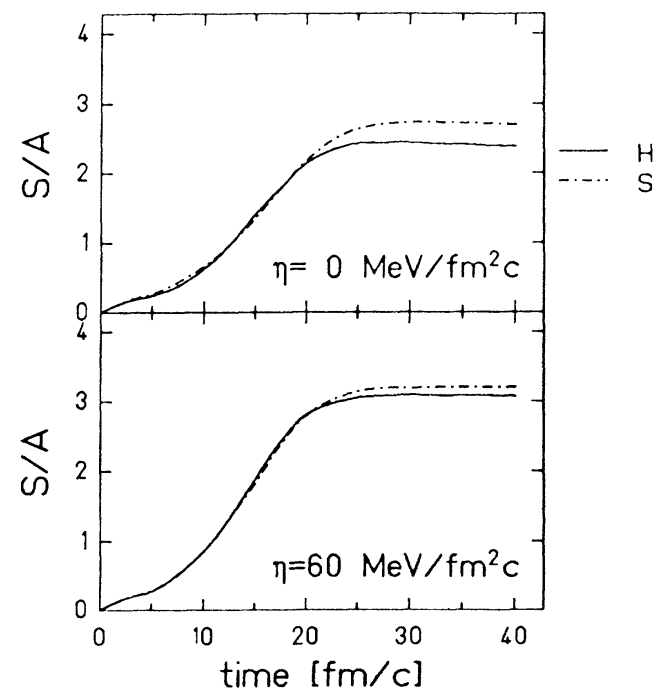

FIG. 3. Influence of viscosity and equation of state on entropy per baryon in a $\mathrm{Au}+\mathrm{Au}$ reaction at $400 \mathrm{MeV} /$ nucleon, impact parameter $b=3 \mathrm{fm}$, for $\eta=0$ (top) and $\eta=60 \mathrm{MeV} / \mathrm{fm}^{2} c$ (bottom): dashed: soft equation of state; straight: hard equation of state.

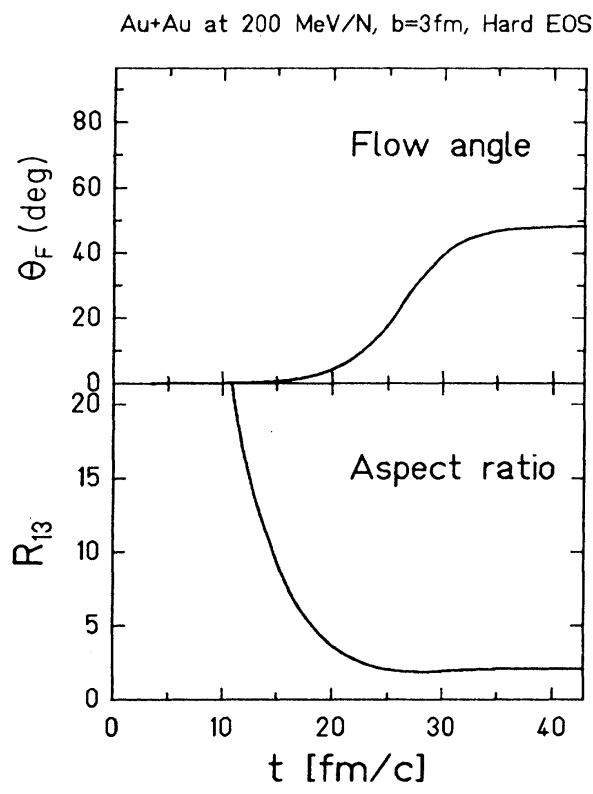

FIG. 4. Time development of flow angle $\theta_{F}$ (top) and aspect ratio $R_{13}$ (bottom) for $\mathrm{Au}+\mathrm{Au}$ at $200 \mathrm{MeV} /$ nucleon, $b=3 \mathrm{fm}$, using a hard equation of state, $\eta=60$ and $\xi=0 \mathrm{MeV} / \mathrm{fm}^{2} c$.

From Figs. 2 and 3 we have seen that the entropy is almost entirely produced during the shock phase of the reaction. Figure 4 shows the time development of the flow angle $\theta_{F}$ [Fig. 4(a)] and the aspect ratio $R_{13}$ [Fig. 4(b)] for the same reaction as in Fig. 2 . The kinetic flow builds up during the expansion phase. Both $\theta_{F}$ and $R_{13}$ saturate asymptotically (after about $30 \mathrm{fm} / c$ ), which gives an indication that the assumption of a fixed breakup time is justified.

To further demonstrate the buildup of kinetic flow, Fig. 5 shows the time development of $d N / d Y$ and $p_{x}$ in the reaction $\mathrm{Au}+\mathrm{Au}$ at $400 \mathrm{MeV} /$ nucleon at $b=3 \mathrm{fm}$ for a hard equation of state, and $\eta=60, \zeta=0 \mathrm{MeV} / \mathrm{fm}^{2} c$. In the beginning, there is of course no transverse momentum at all, while the incident nuclei are centered around $Y_{\text {proj }}$ and $Y_{\text {targ }}$, respectively. Then, more and more nucleons are stopped, which can be seen from the $d N / d Y$ distribution, exhibiting a broad maximum around $Y=Y_{\text {c.m. }}$. The transverse flow is developing simultaneously with the stopping.

From the final $d N / d Y$ distribution one can see that most of the emitted nucleons have little longitudinal momentum in the c.m. system. This means that the $p_{x}$ observable does not carry the most interesting information about the densest matter, since $p_{x}$ vanishes per definition for those nucleons which have experienced the most violent stopping ( $Y=Y_{\text {c.m. }}$ ) during the collision. Also, for exactly central collisions, which show the strongest flow, $p_{x} \equiv 0$ everywhere.

Note that the $p_{x}$ distribution from Fig. 5 has been computed from the average collective hydrodynamic density and momentum per cell, without chemical breakup and thermal evaporation. It should not be compared directly with experimental $p_{x}$ distributions, because the 


\section{Au+Au at $200 \mathrm{Mev} / \mathrm{N}, b=3 \mathrm{fm}$, Hard EOS, $\eta=60 \mathrm{MeV} / \mathrm{fm}^{2} \mathrm{c}$}

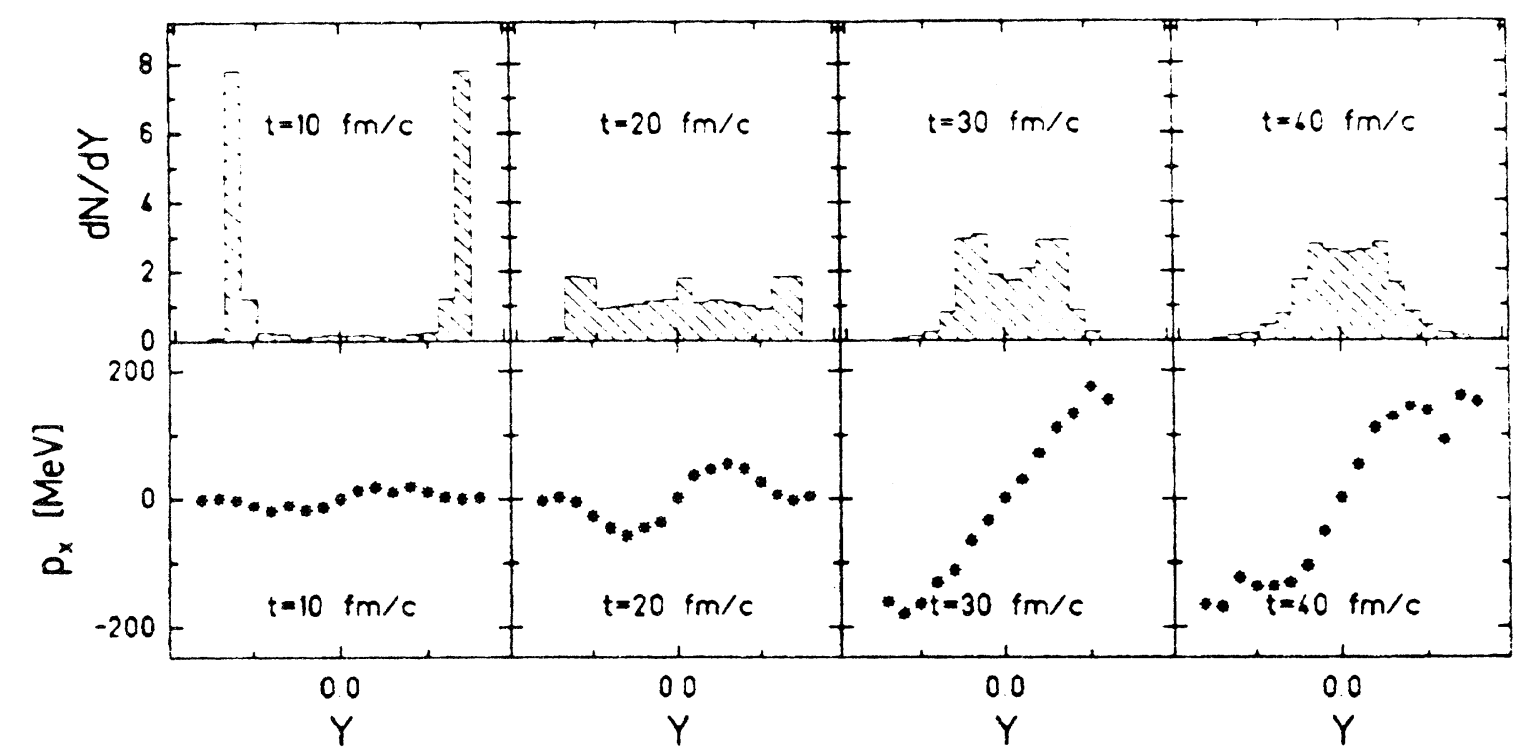

FIG. 5. Time development of $d N / d Y$ (top) and $p_{x} / A$ (bottom) for $\mathrm{Au}+\mathrm{Au}$ at $200 \mathrm{MeV} / \mathrm{nucleon}, b=3 \mathrm{fm}$, using a hard equation of state; $\eta=60$ and $\xi=0 \mathrm{MeV} / \mathrm{fm}^{2} c$.

microscopic "thermal" momentum distribution within each cell has not been taken into account. It will be included in Sec. V D.

\section{B. Entropy}

Figure 6 compares entropy data from Plastic Ball experiments with fluid-dynamics calculations. The excitation function of the entropy is shown for central collisions $(b=1 \mathrm{fm})$ of $\mathrm{Au}+\mathrm{Au}$ [Fig. 6(a)] and $\mathrm{Nb}+\mathrm{Nb}$ [Fig. 6(b)]. Note that the difference between soft and hard equations of state is small as compared to the influence of viscosity.

It can be seen that the nonrelativistic model yields satisfactory results, if the viscosity is introduced $(\eta=60$ $\mathrm{MeV} / \mathrm{fm}^{2} \mathrm{c}$ ). One is led to the conclusion that the nuclear viscosity is of the order of $\eta \approx 60 \mathrm{MeV} / \mathrm{fm}^{2} c$; these values are in agreement with the theoretical result of Danielewicz [12], which has been derived for infinite matter from the Uehling-Uhlenbeck equation, and with recent calculations of Schürmann [24].

\section{Flow angle and aspect ratio}

In this section we want to study some properties of the flow tensor, which can be computed from hydrodynamic densities and momentum distributions. Neither $\theta_{F}$ nor $R_{13}$ can be measured experimentally because of the fluctuations imposed by finite multiplicities. Only the Jacobian flow angle distribution $d N / d \cos \theta$ was considered measurable so far [25]. To compare nuclear fluiddynamics calculations with experimental data, one therefore had to compute $d N / d \cos \theta$ distributions by random- ly generating numerous "events" with finite experimental multiplicities and integrating over a range of impact parameters [5]. This method was applied successfully to experimental $d N / d \cos \theta$ data measured by the Plastic Ball collaboration for the system $\mathrm{Nb}+\mathrm{Nb}$ at 400

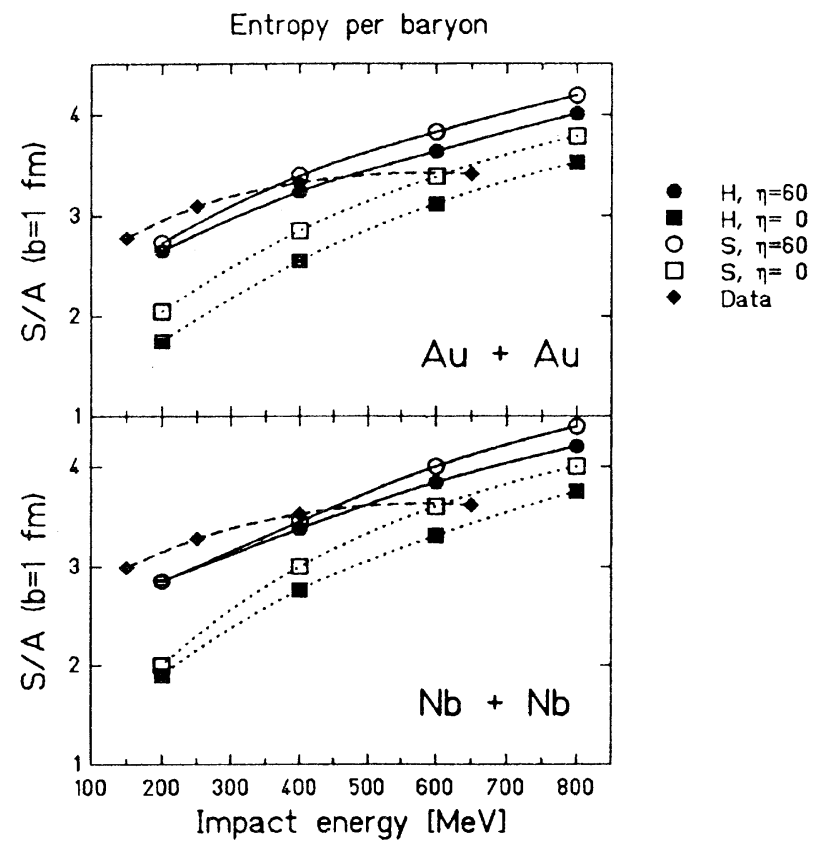

FIG. 6. Excitation function of the entropy per baryon for central collisions $(b=1 \mathrm{fm})$ of $\mathrm{Au}+\mathrm{Au}$ (top) and $\mathrm{Nb}+\mathrm{Nb}$ (bottom). Experimental data are for the fifth multiplicity bin. 


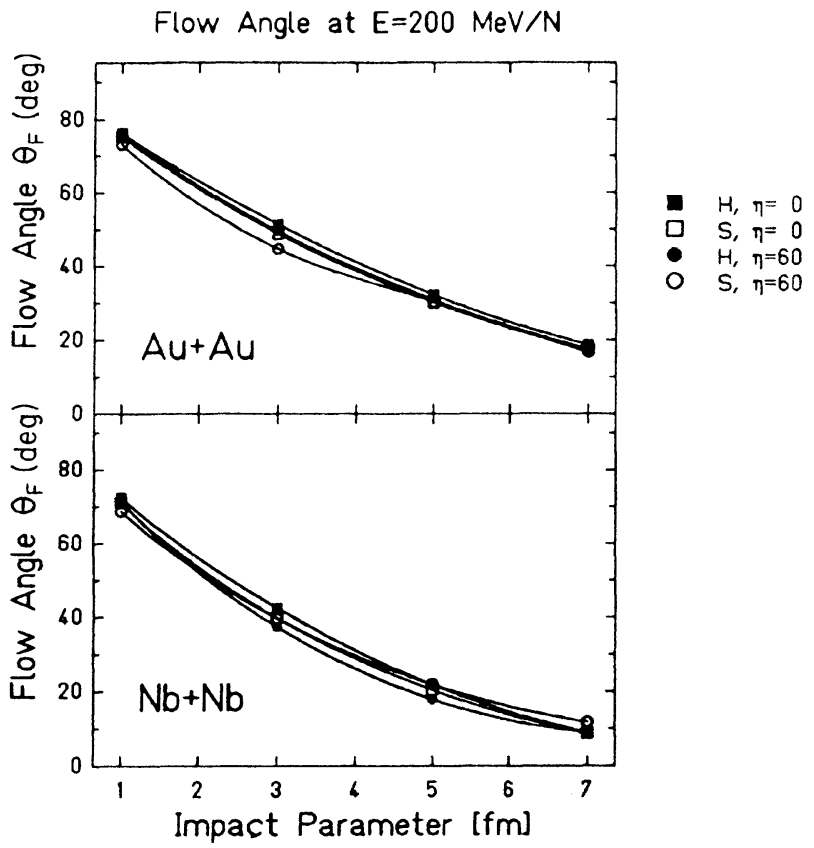

FIG. 7. Flow angle $\theta_{F}$ as a function of impact parameter in $\mathrm{Au}+\mathrm{Au}$ (top) and $\mathrm{Nb}+\mathrm{Nb}$ collisions (bottom).

\section{$\mathrm{MeV} /$ nucleon.}

Here we do not want to repeat this procedure and abandon the direct comparison of our calculation with experiment. Instead, we focus on the pure averaged collective hydrodynamic momentum distribution neglecting again the microscopic "thermal" momentum distribution within each cell. In this way, flow angle and aspect ratio can be calculated exactly for each system. Hence we can

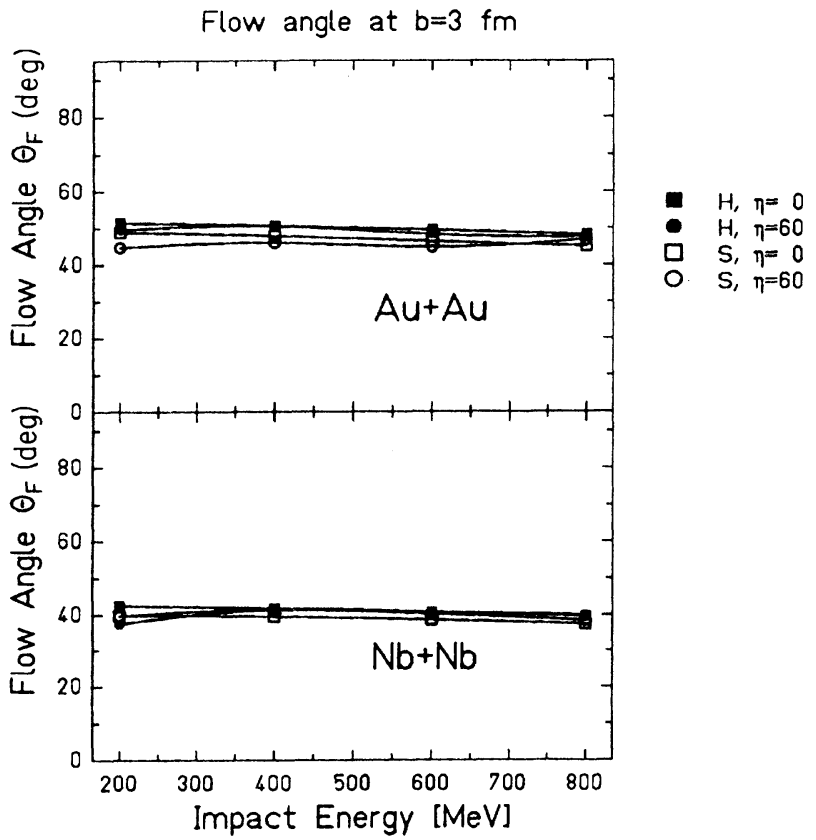

FIG. 8. Excitation function of the flow angle $\theta_{F}$ in $\mathrm{Au}+\mathrm{Au}$ (top) and $\mathrm{Nb}+\mathrm{Nb}$ collisions (bottom). display $\theta_{F}$ as a function of impact parameter, viscosity, equation of state, and bombarding energy.

It turns out that the pure collective flow angle is almost independent of both the equation of state, the viscosity [13], and bombarding energies, as shown in Fig. 7. It does, however, depend very strongly on the impact parameter, as can be seen from Fig. 8. This implies that the pure collective flow angle is a geometric quantity. Its scaling properties are given by $b / R$ or $b / A^{1 / 3}$, respectively. The experimentally observable flow angle, however, needs to be computed from the superposition of the purely collective flow and the microscopic (thermal) motion of the emitted particles. The latter are, however, quite sensitive to the dynamics, as we will now show.

\section{Collective transverse momentum transfer}

The transverse momentum transfer $p_{x} / A$ has been measured experimentally for the systems $\mathrm{Ca}+\mathrm{Ca}, \mathrm{Nb}$ $+\mathrm{Nb}$, and $\mathrm{Au}+\mathrm{Au}$ at various bombarding energies. Data have been selected according to charge and multiplicity [22,23].

Again there are two different ways to calculate $p_{x} / A$ in nuclear fluid dynamics.

(1) It can be computed from the average $\delta$ collective momentum for every cell in the nuclear fluid and plotted as a function of rapidity (purely macroscopic distribution), such that the "thermal" momentum distribution is neglected.

(2) It can be calculated by integration of $(1 / p) d^{3} \sigma / d E d \Omega d \phi$ (microscopic distribution) which is obtained after the microscopic "thermal" distribution is added to the purely collective motion. Different results are obtained for different particle species, which can then be compared with experiment. We want to emphasize that there is little dependence on the breakup time, if the breakup condition $\rho_{\max }<\rho_{0}$ is fulfilled [14,21].

Figure 9 provides an overview of the macroscopic $p_{x}$ distribution obtained for various collisions of $\mathrm{Au}+\mathrm{Au}$ at $200 \mathrm{MeV} /$ nucleon. All calculations have been done with the hard equation of state. The impact parameter $b$ varies from left to right $(b=1,3,5$, and $7 \mathrm{fm})$ and the viscosity $\eta$ varies from top to bottom $(\eta=0,30$, and 60 $\mathrm{MeV} / \mathrm{fm}^{2} \mathrm{c}$ ). The influence of evaporation has been neglected. As one can clearly see, the collective flow, which is taken to be $d p_{x} /\left.d Y\right|_{Y_{p}}$, decreases for increasing impact parameter and viscosity. Note that the maximum flow does not occur at intermediate impact parameters, but for rather central collisions ( $b=1 \mathrm{fm}$ ), which show a collective flow of more than $300 \mathrm{MeV} / \mathrm{c}^{2}$. On the other hand, $p_{x}$ vanishes per definition, if $b=0$. Therefore, the macroscopic $p_{x}$ distribution as computed from the collective momenta and densities, i.e., without the addition of the "thermal" momentum distribution in each cell, does not reproduce the experimentally observed multiplicity dependence, where the maximum in $p_{x}\left(\boldsymbol{M}_{c}\right)$ occurs in the fourth multiplicity bin [23], corresponding to $b \approx 3$ fm. It also overestimates the magnitude of the flow by a factor of 2 or more as compared with data.

Figure 10(a) demonstrates how the macroscopic hydrodynamic $p_{x} / A$ is affected by the evaporation: The flow 


\section{$\mathrm{Au}+\mathrm{Au}$ at $200 \mathrm{MeV} / \mathrm{N}$, Hard EOS, $b=1,3,5,7 \mathrm{fm}$ hor., $\eta=0,30,60 \mathrm{MEV}$ ver.}

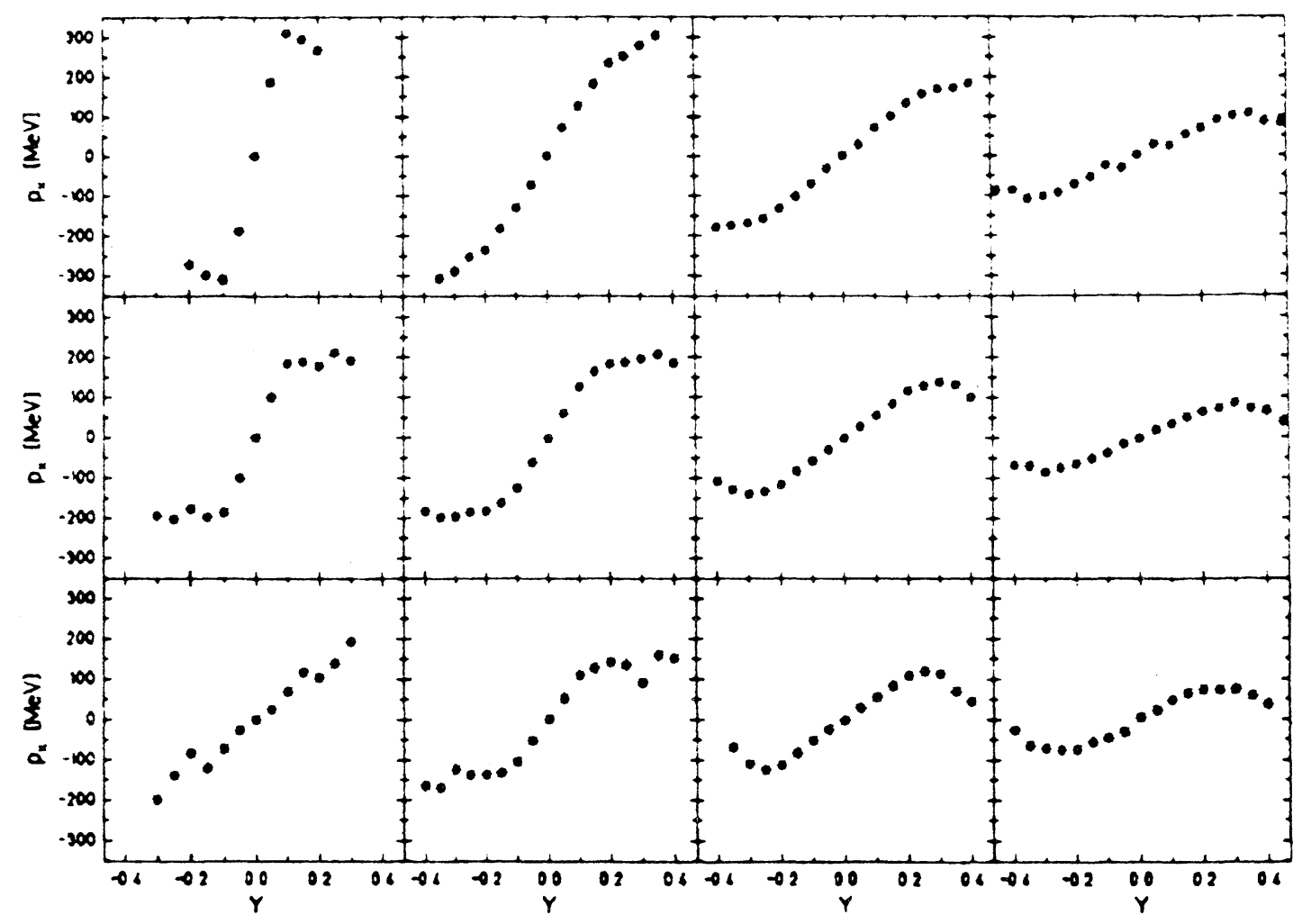

FIG. 9. Macroscopic $p_{x}$ distribution for various reactions of $\mathrm{Au}+\mathrm{Au}$ at $200 \mathrm{MeV} /$ nucleons, using the hard equation of state. The impact parameter varies from left to right $(b=1,3,5$, and $7 \mathrm{fm}$ ), shear viscosity $\eta$ from top to bottom ( $\eta=0,30$, and 60 $\mathrm{MeV} / \mathrm{fm}^{2} \mathrm{c}$.

decreases drastically for protons, whereas ${ }^{4} \mathrm{He}$ (which, in the present model, in fact, also represents all heavier fragments) still shows the strong collective flow which is predicted by the purely macroscopic distribution. The calculation has been done for $\mathrm{Au}+\mathrm{Au}$ at 200 $\mathrm{MeV} /$ nucleon and $b=3 \mathrm{fm}$, using the hard equation of state and $\eta=60 \mathrm{MeV} / \mathrm{fm}^{2} c$.

To show the influence of viscosity and equation of state on $p_{x} / A$ we include Fig. 10(b), which compares the proton $p_{x}$ for soft and hard equations of state with $\eta=0$ and $60 \mathrm{MeV} / \mathrm{fm}^{2} c$. It can be seen that the equation of state has a $20 \%$ influence for a viscous calculation, due to the small maximum compression $\left(\rho_{\max } / \rho_{0} \approx 1.5\right)$ achievable at these low energies.

In Fig. 11 we compare our calculation (hard equation of state, $\eta=60 \mathrm{MeV} / \mathrm{fm}^{2} c$ ) with experimental data for (a) $Z=1$ and (b) $Z=2$. There is a remarkable quantitative agreement with experimental data. The discrepancies at $Y<0$ are due to efficiency cuts of the Plastic Ball detector at target rapidity, which have been neglected in our calculation.

This figure gives another clear evidence for a fairly high viscosity of $\eta \approx 60 \mathrm{MeV} / \mathrm{fm}^{2} c$. Soft and hard equations of state give about $20 \%$ differences in the $p_{x}$ distri- bution at this viscosity, while a factor of 2 change in the viscosity would give larger differences. Hence, both the equation of state and nonequilibrium effects influence the $p_{x}$ distributions, which can therefore not be used alone to pin down one or the other from the data.

\section{E. Azimuthal angular correlation}

Following the approach of Kampert [23] and Doss et al. [26], we studied the dimensionless quantity $p_{x} /\left|p_{\perp}\right|$. Figure 12(a) shows the strong correlation of hydrodynamic flow which is preserved by heavier fragments. Evaporation of light particles, however, leads to a much more isotropic flow.

Once again it turns out that collective flow is very sensitive to the viscosity: Figure 12(b) shows that the angular correlation of protons in a viscous calculation of $\mathrm{Au}$ $+\mathrm{Au}$ at $200 \mathrm{MeV} /$ nucleon is roughly $40 \%$ less than for the nonviscous case. Note the small $(20 \%)$ influence of the equation of state in both calculations.

Figure 13 shows that the angular correlation is overestimated by nuclear fluid dynamics for both $Z=1$ [Fig. 13(a)] and $Z=2$ fragments [Fig. 13(b)]. 
$\mathrm{Au}+\mathrm{Au}$ at $200 \mathrm{MeV} / \mathrm{N}, \mathrm{b}=3 \mathrm{fm}$, Hard EOS, $\eta=60 \mathrm{MeV} / \mathrm{fm}^{2}$

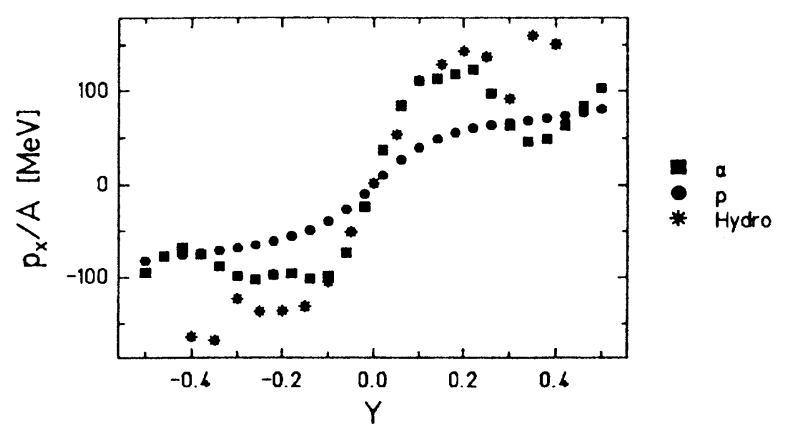

$\mathrm{Au}+\mathrm{Au}$ at $200 \mathrm{MeV} / \mathrm{N}, \mathrm{b}=3 \mathrm{fm}$, proton $p_{x}$

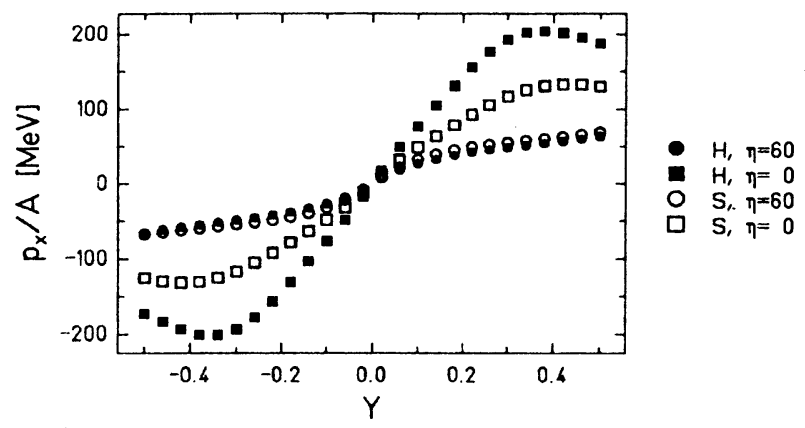

FIG. 10. Top: $p_{x}$ distribution in a $\mathrm{Au}+\mathrm{Au}$ reaction at 200 $\mathrm{MeV} /$ nucleon, $b=3 \mathrm{fm}$, hard equation of state, $\eta=60, \xi=0$ $\mathrm{MeV} / \mathrm{fm}^{2} c$, comparing macroscopic (hydro) and microscopic $(p, \alpha)$ results. Bottom: microscopic $p_{x}$ distribution of protons in $\mathrm{Au}+\mathrm{Au}$ at $b=3 \mathrm{fm}$, hard and soft equations of state, nonviscous $(\eta=0)$ and viscous $\left(\eta=60 \mathrm{MeV} / \mathrm{fm}^{2} c\right)$ calculation.

$A u+A u$ at $200 \mathrm{MeV} / \mathrm{N}, b=3 \mathrm{fm}$, Hard EOS, $\eta=60 \mathrm{MeV} / \mathrm{fm}^{2}$

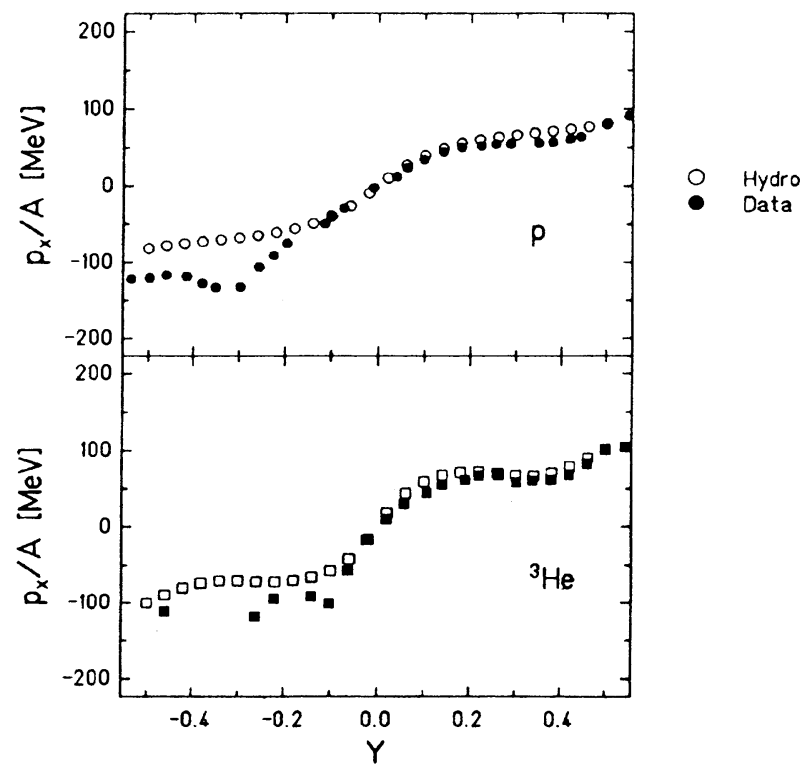

FIG. 11. $p_{x}$ distribution in a $\mathrm{Au}+\mathrm{Au}$ reaction at 200 $\mathrm{MeV} /$ nucleon, $b=3 \mathrm{fm}$, hard equation of state, $\eta=60, \xi=0$ $\mathrm{MeV} / \mathrm{fm}^{2} c$, comparing theory and experiment for $Z=1$ (top) and $Z=2$ (bottom).
$A u+A u$ at $200 \mathrm{MeV} / \mathrm{N}, b=3 \mathrm{fm}, \mathrm{Hard} E O S, \eta=60 \mathrm{MeV} / \mathrm{fm}^{2}$

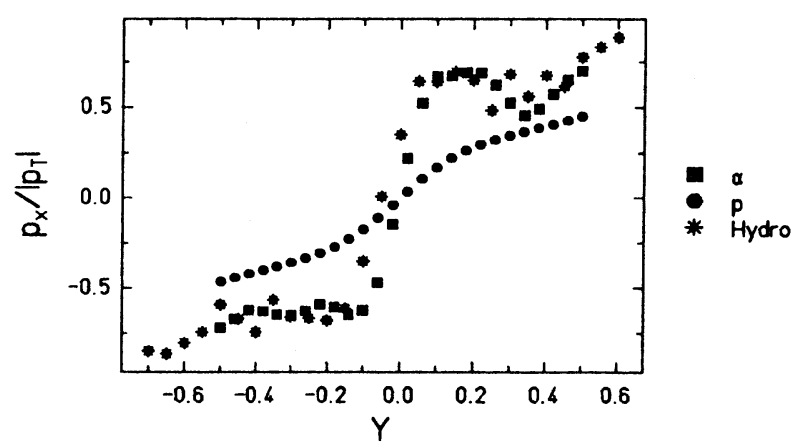

$A u+A u$ at $200 \mathrm{MeV} / \mathrm{N}, b=3 \mathrm{fm}$, proton $p_{x} /\left|p_{T}\right|$

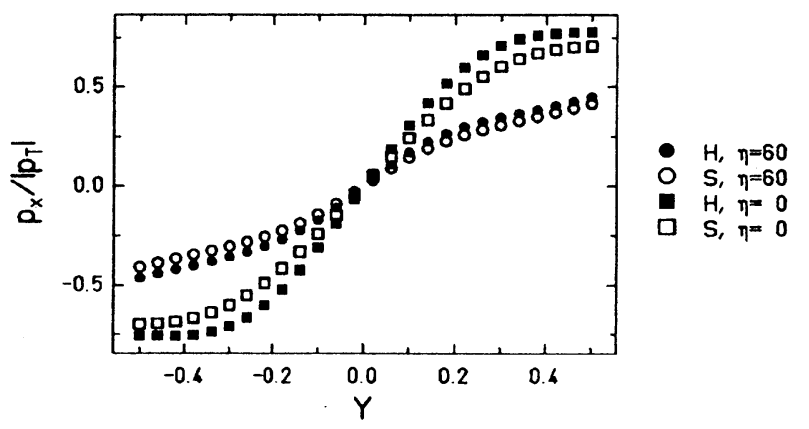

FIG. 12. Top: $p_{x} /\left|p_{1}\right|$ distribution in a $\mathrm{Au}+\mathrm{Au}$ reaction at $200 \mathrm{MeV} /$ nucleon, $b=3 \mathrm{fm}$, hard equation of state, $\eta=60, \zeta=0$ $\mathrm{MeV} / \mathrm{fm}^{2} c$, comparing macroscopic (hydro) and microscopic $(p, \alpha)$ results. Bottom: microscopic $p_{x} /\left|p_{1}\right|$ distribution of protons in $\mathrm{Au}+\mathrm{Au}$ at $b=3 \mathrm{fm}$, hard and soft equations of state, nonviscous $(\eta=0)$ and viscous $\left(\eta=60 \mathrm{MeV} / \mathrm{fm}^{2} c\right)$ calculation.

$\mathrm{Au}+\mathrm{Au}$ at $200 \mathrm{MeV} / \mathrm{N}, b=3 \mathrm{fm}, \mathrm{Hard}$ EOS, $\eta=60 \mathrm{MeV} / \mathrm{fm}^{2}$

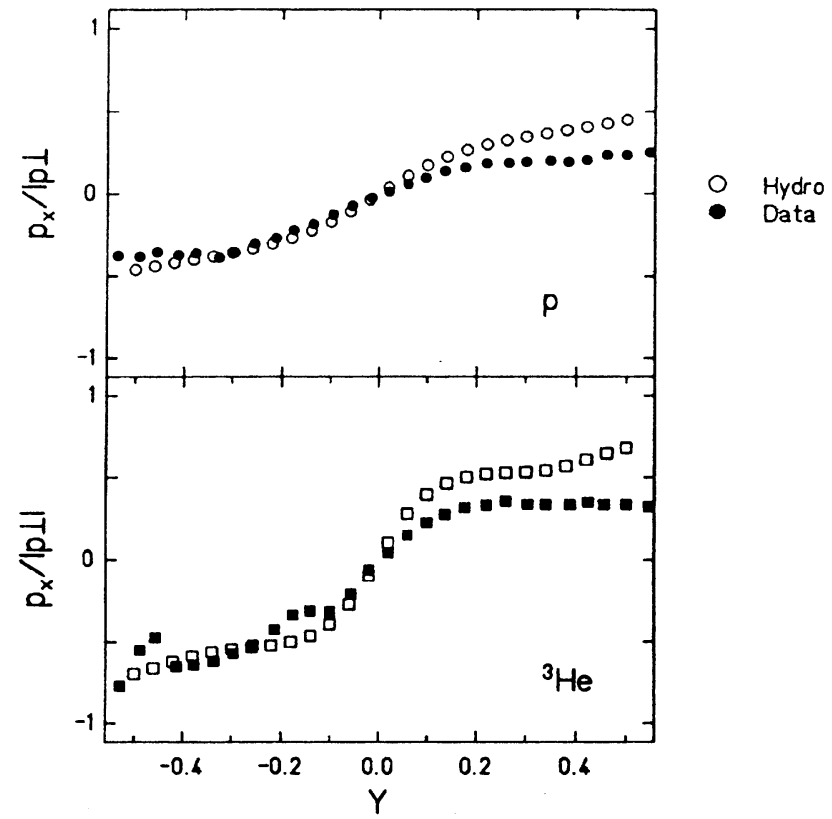

FIG. 13. $p_{x} /\left|p_{1}\right|$ distribution in a $\mathrm{Au}+\mathrm{Au}$ reaction at 200 $\mathrm{MeV} /$ nucleon, $b=3 \mathrm{fm}$, hard equation of state, $\eta=60, \xi=0$ $\mathrm{MeV} / \mathrm{fm}^{2} c$, comparing theory and experiment for $Z=1$ (top) and $Z=2$ (bottom). 


\section{F. Angular rapidity distribution}

Besides the collective in-plane flow discussed above there is also a completely independent collective effect, namely, the off-plane $\left(\phi=90^{\circ}\right)$ squeeze out at $Y_{\text {c.m. }}=0$, which was predicted by hydrodynamics $[4,5]$ and recently discovered experimentally $[8,19]$.

To provide for a synopsis of this effect, we have included Fig. 14 which displays $d N /\left.d Y d \phi\right|_{Y=Y_{p}}$ [Fig. 14(a)] and $d N /\left.d Y d \phi\right|_{Y=Y_{\text {c.m. }}}$ [Fig. 14(b)] for different viscosities $\left(\eta=0,30\right.$, and $\left.60 \mathrm{MeV} / \mathrm{fm}^{2} c\right)$. The peak-to-valley ratio decreases drastically as viscosity increases.

How does this effect depend on the equation of state? Figure 15 shows $d N /\left.d Y d \phi\right|_{Y=0}$ for the soft [Fig. 14(a)] and the hard equations of state [Fig. 14(b)] for different viscosities $\eta$ simultaneously. Experimental data from Kampert [19] have been included in the figure. It can be seen that the soft equation of state can reproduce the data qualitatively, if $\eta \approx 30 \mathrm{MeV} / \mathrm{fm}^{2} c$. For the hard equation of state, a viscosity of $\eta \approx 50 \mathrm{MeV} / \mathrm{fm}^{2} c$ is needed. However, $\eta=30 \mathrm{MeV} / \mathrm{fm}^{2} c$ cannot account consistently for the observed entropy and transverse momentum values.

Figure 16 displays the triple-differential cross section of protons with a kinetic energy in the c.m. frame of $T=180 \mathrm{MeV}$ in a $\mathrm{Au}+\mathrm{Au}$ reaction at 400 $\mathrm{MeV} /$ nucleon bombarding energy for impact parameters $b=1,3,5$, and $7 \mathrm{fm}$. The calculation was done using the hard equation of state and a shear viscosity of $\eta=60$ $\mathrm{MeV} / \mathrm{fm}^{2} c$. The upper picture shows the in-plane cross section at $\phi=0^{\circ}$, and the lower one the out-of-plane cross section at $\phi=90^{\circ}$.

The in-plane cross section shows maxima at $\theta=80^{\circ}$, $50^{\circ}, 30^{\circ}$, and $10^{\circ}$ for $b=1,3,5$, and $7 \mathrm{fm}$. As can be seen from Fig. 7, this corresponds to the flow angle at these impact parameters, respectively.

On the other hand, the out-of-plane $\left(\phi=90^{\circ}\right)$ cross section has its maximum at forward and/or backward polar angles, $\theta=0^{\circ}$ and $180^{\circ}$, except for very central collisions at $b=1 \mathrm{fm}$. This affects the $\phi$-integrated (doubledifferential) cross sections shown in Fig. 17. There is a

\section{$\mathrm{Au}+\mathrm{Au}$ at $400 \mathrm{MeV} / \mathrm{N}, \mathrm{b}=3 \mathrm{fm}$, Hard EOS}

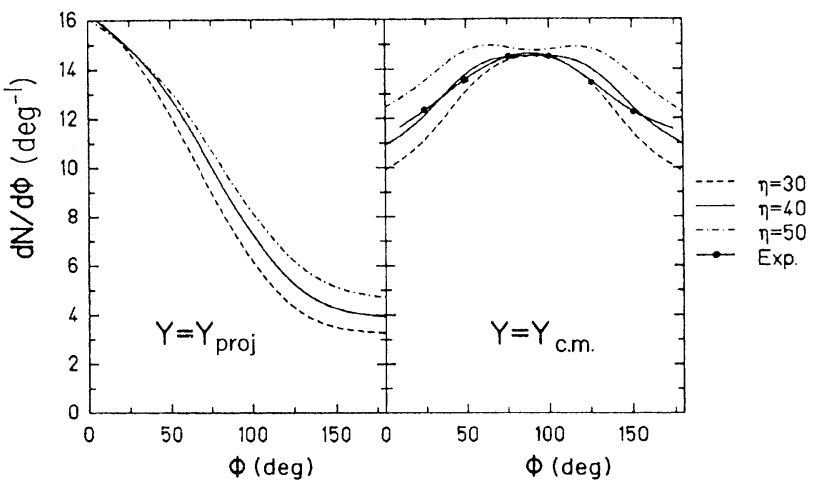

FIG. 14. $d N / d Y d \phi$ distribution in a $\mathrm{Au}+\mathrm{Au}$ reaction at $400 \mathrm{MeV} /$ nucleon, $b=3 \mathrm{fm}$, hard equation of state, $\eta=40$ $\mathrm{MeV} / \mathrm{fm}^{2} c$, cuts at $Y=Y_{\text {proj }}$ (left) and $Y=Y_{\text {c.m. }}$ (right).

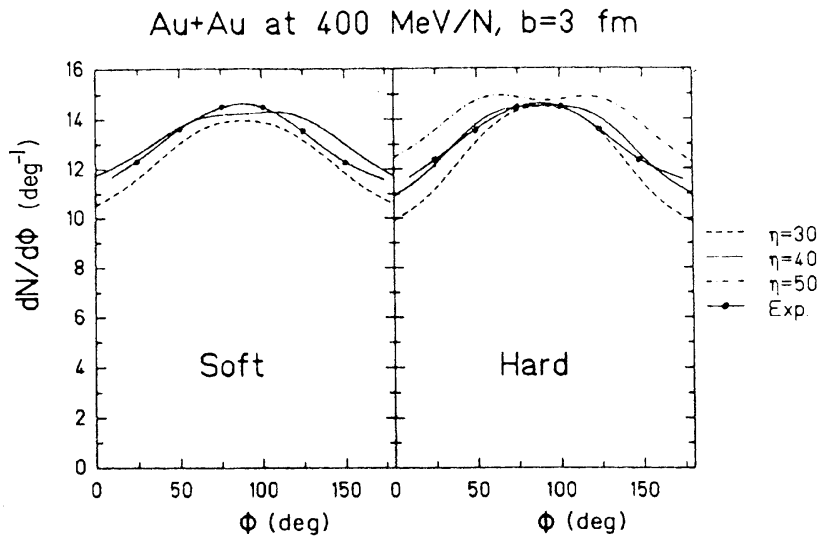

FIG. 15. $d N / d Y d \phi$ distribution in a $\mathrm{Au}+\mathrm{Au}$ reaction at $400 \mathrm{MeV} /$ nucleon, $b=3 \mathrm{fm}, \eta=40 \mathrm{MeV} / \mathrm{fm}^{2} c$, cuts at $Y=Y_{\text {c.m. }}$, soft (top) and hard (bottom) equations of state, compared with data.

strong peak at $\theta=90^{\circ}$ for central collisions, which can be seen for both $200 \mathrm{MeV} /$ nucleon [Fig. 17(a)] and 400 $\mathrm{MeV} /$ nucleon [Fig. 17(b)] bombarding energy. No corresponding peak can be observed in more peripheral collisions. This holds for all combinations of equation of state and viscosity in the reaction systems $\mathrm{Au}+\mathrm{Au}$ and $\mathrm{Nb}+\mathrm{Nb}$ which we investigated.

Because of the magnitude of the effect we may conclude that the double-differential cross sections must exhibit a strong $90^{\circ}$ enhancement in the proton spectra, if

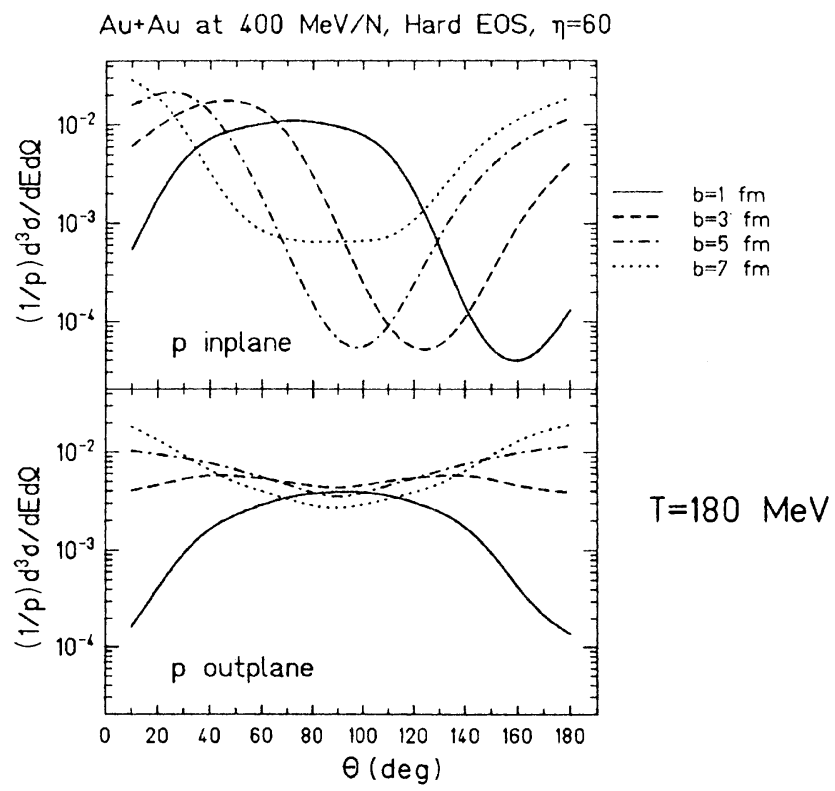

FIG. 16. Triple-differential invariant cross section for 180 $\mathrm{MeV}$ protons in-plane (upper picture) and out-of-plane (lower picture) in $\mathrm{Au}+\mathrm{Au}$ at $400 \mathrm{MeV} /$ nucleon for different impact parameters $b=1,3,5$, and $7 \mathrm{fm}$. Hard equation of state, $\eta=60$ $\mathrm{MeV} / \mathrm{fm}^{2} c$. The cross sections are measured in units of $\mathrm{mb} / \mathrm{MeV} \mathrm{sr}$. 


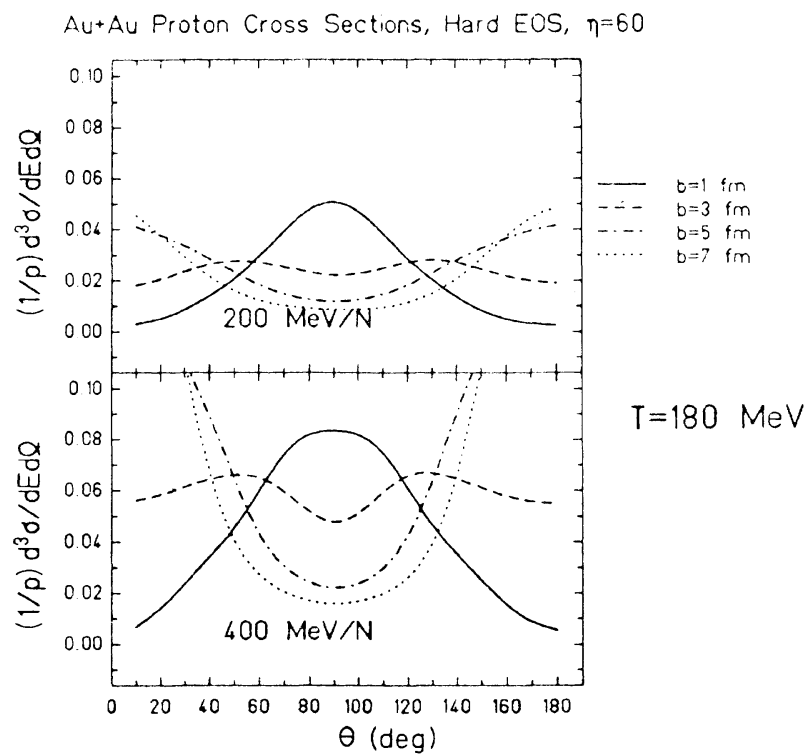

FIG. 17. Double-differential invariant cross section for 180 $\mathrm{MeV}$ protons (azimuthally averaged) in $\mathrm{Au}+\mathrm{Au}$ at 200 $\mathrm{MeV} /$ nucleon (upper picture) and $400 \mathrm{MeV} /$ nucleon (lower picture) for different impact parameters $b=1,3,5$, and $7 \mathrm{fm}$. Hard equation of state, $\eta=60 \mathrm{MeV} / \mathrm{fm}^{2} c$.

only very central collisions are taken into account. This can be done experimentally by a very rigid multiplicity selection.

Recently Claesson et al. [9] have performed such an analysis for the system $\mathrm{La}+\mathrm{La}$ at $246 \mathrm{MeV} /$ nucleon. The enhancement can also be observed in Fig. 18, show-

$\mathrm{Au}+\mathrm{Au}$ at $200 \mathrm{MeV} / \mathrm{N}$, Hard EOS, $\eta=60 \mathrm{MeV} / \mathrm{fm}^{2}$

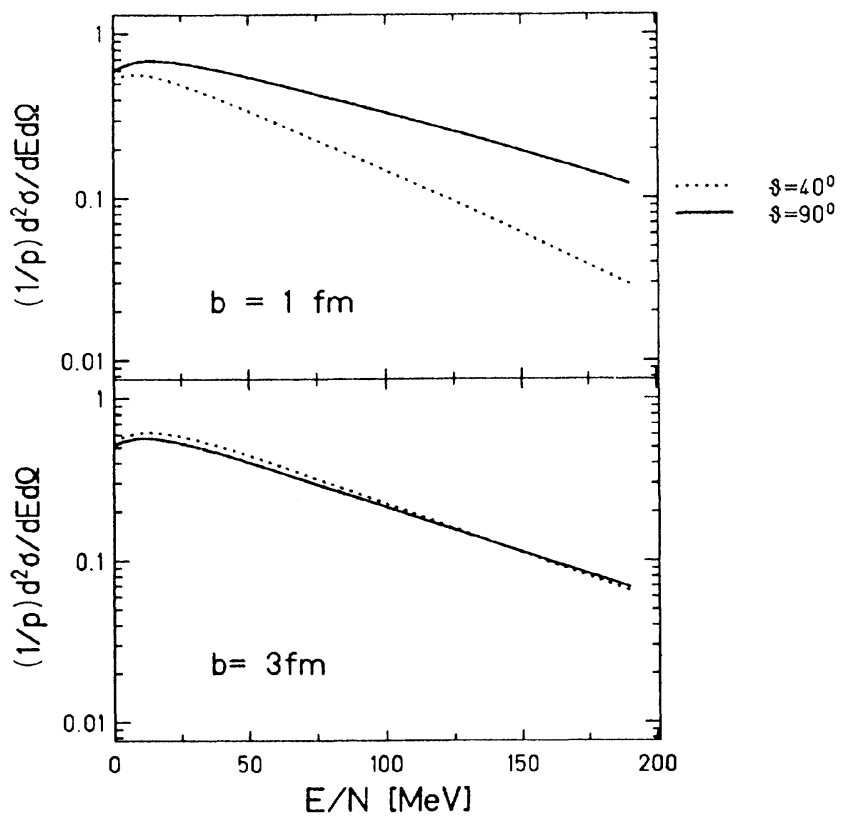

FIG. 18. Azimuthally averaged photon spectra for $\theta=40^{\circ}$ (dotted) and $\theta=90^{\circ}$ (straight lines) in $\mathrm{Au}+\mathrm{Au}$ at 200 $\mathrm{MeV} /$ nucleon, $b=1 \mathrm{fm}$ (upper picture), and $b=3 \mathrm{fm}$ (lower picture). Hard equation of state, $\eta=60 \mathrm{MeV} / \mathrm{fm}^{2} c$. ing the proton spectra as predicted by nuclear fluid dynamics for the reaction $\mathrm{Au}+\mathrm{Au}$ at $200 \mathrm{MeV} /$ nucleon, using the hard equation of state and $\eta=60 \mathrm{MeV} / \mathrm{fm}^{2} c$ at two different c.m. angles, $\theta=40^{\circ}$ and $90^{\circ}$. In Fig. 18(a) at $b=1 \mathrm{fm}$ the $90^{\circ}$ spectrum is considerably higher than that at $40^{\circ}$, whereas in Fig. 18(b) (at $\left.b=3 \mathrm{fm}\right)$ they are roughly the same. Depending on the proton energy, the $\theta=90^{\circ}$ enhancement can be a factor of 2 or more as compared to the $\theta=40^{\circ}$ spectra in central collisions. This shows the rather violent stopping power of nuclear matter at these energies and the strongly correlated sideward motion of compressed nuclear matter.

Recently the European $4 \pi$ collaboration [28] has devised a new method to isolate the most central reactions with impact parameters less than about $1 \mathrm{fm}$ : the idea is the isolation of azimuthally symmetric events with high multiplicity, i.e, the exclusion of intermediate impact parameters of $b=3-5 \mathrm{fm}$ by using the absence of the bounce-off and directed $\mathbf{p}_{x}$ flow, which are zero by definition in very central events (although the transverse flow in $p_{\perp}$ is maximal there). Indeed, the European $4 \pi$ collaboration has successfully isolated these long-sought events. They have discovered that these rare events do indeed exhibit clear flow for all fragments (light and heavy) with very few particles left at low $p_{\perp}$ values along the beam axis, and a nearly completely depopulated projectile rapidity region. Figure 19 shows the corresponding predictions of our viscous hydrodynamic model for protons and "alphas" (as mentioned, the latter include all complex fragments with $A \geq 4$ in the present code). Note that the width of the rapidity distribution depends sensitively on the viscosity, which will be an important independent check for our $\eta$ values given above. We would like to add that similar conclusions have been reached on the basis of QMD calculations [27].

\section{CONCLUSIONS}

We are led to the following conclusions from the comparison of our calculations with Plastic Ball data:

(a) Viscous nuclear hydrodynamics can quantitatively reproduce heavy-ion data. We have shown that several observables, namely, the entropy per baryon $S / A$, the $p_{x}$ distributions from transverse momentum analysis, tripledifferential cross sections $1 / p \partial^{3} \sigma / \partial E \partial \Omega \partial \phi$ and rapidity distributions $d N / d Y d \phi$ can be calculated in good quantitative agreement with experimental data. It is, however, necessary to treat the chemical breakup separately, using a quantum statistical model, which takes into account the microscopic evaporation of fragments. Hydrodynamics without evaporation strongly overestimates the collective motion of light fragments, which are affected by their thermal momentum distribution.

(b) Nuclear hydrodynamics shows a strong sensitivity to the nuclear shear viscosity. Both the entropy production and the kinetic flow observed in heavy-ion reactions cannot be explained in terms of ideal hydrodynamics. It turns out that the nonequilibrium properties of nuclear matter, described by the transport coefficients, play an important role in the transition to equilibrium. Calculations with a constant coefficient of viscosity - neglecting 


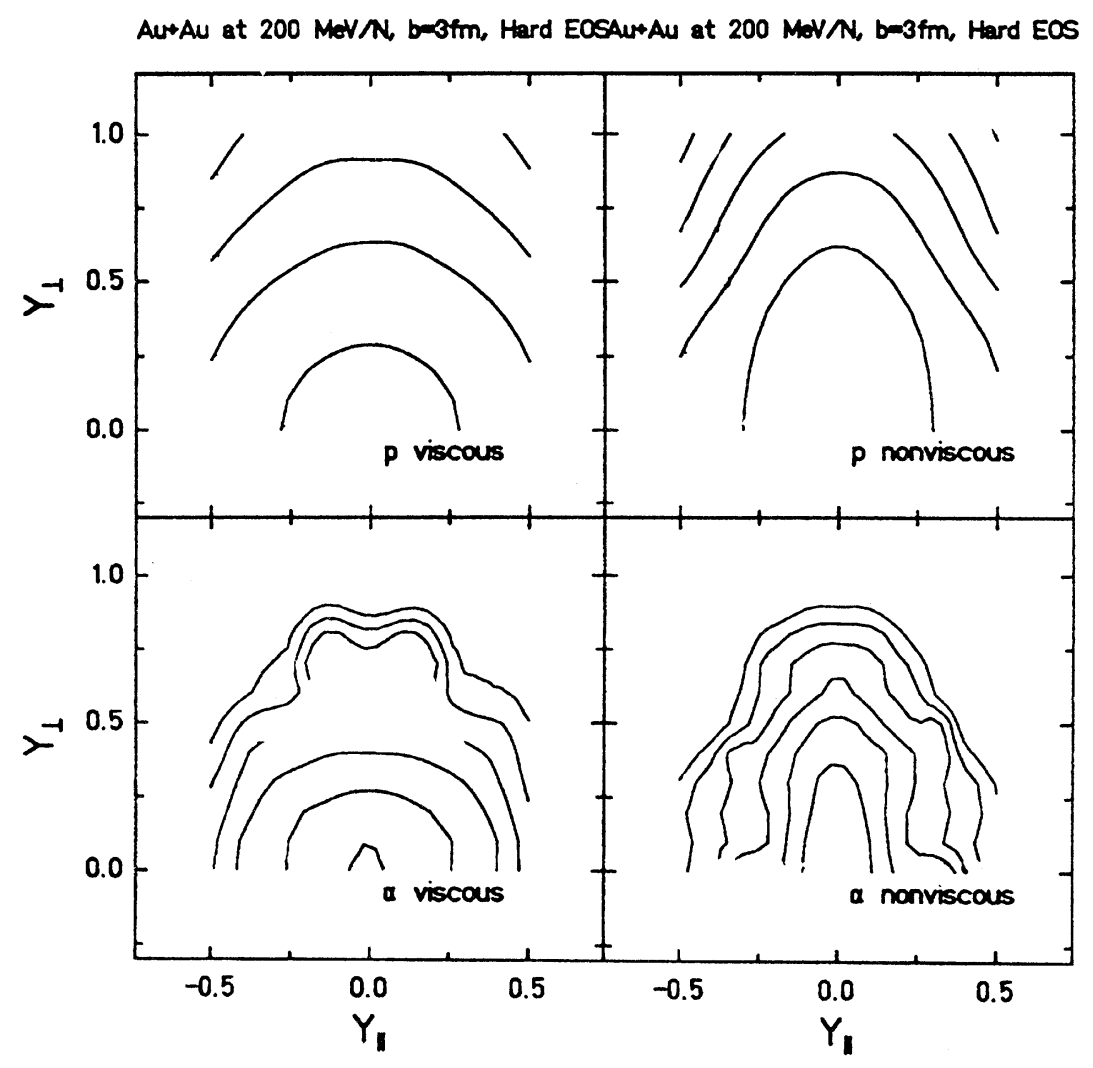

FIG. 19. Double-differential invariant proton (upper) and "alpha" particle distributions in the $y_{\perp}$ and $y_{\|}$planes. For the inviscid case (right-hand side) the distributions are much more narrow and the $p_{1}$ values much larger than for the viscous calculations (lefthand side). This offers an independent measurement of viscosity from new data by the European $4 \pi$ collaboration at GSI.

thermal conductivity - provide an upper bound of $\eta \approx 60$ $\mathrm{MeV} / \mathrm{fm}^{2} c$, which can be obtained from entropy and transverse momentum analysis. This is slightly higher than in microscopic calculations, where one gets $\eta \approx 40-50 \mathrm{MeV} / \mathrm{fm}^{2} c[12,24,27]$. Part of the entropy production may be due to the bulk viscosity $\zeta$, which does not influence kinetic flow. Therefore, in principle, it should be possible to fix the bulk viscosity - respectively, the time of chemical breakup-from entropy data. So far, we have not been able to do so, since this task requires the study of excitation functions up to 800 $\mathrm{MeV} /$ nucleon which is not reliable in our nonrelativistic model.

(c) The nuclear equation of state could only be extracted together with the viscosity from a simultaneous description of the triple-differential cross sections and rapidity distributions. None of the observables discussed so far depend solely on the equation of state. Only if sufficiently precise information on the nonequilibrium viscous effects is obtained from the data may we hope to pin down the nuclear equation of state.

In conclusion, we have demonstrated that viscous nuclear fluid dynamics can quantitatively explain highmultiplicity triggered central heavy-ion data. Both the predicted bounce-off in the reaction plane as well as the squeeze out of dense matter perpendicular to the reaction plane agree with $4 \pi$ data. It is shown that very central collisions exhibit the preferential $90^{\circ}$ flow even in doubledifferential proton spectra. This conclusion seems to be in accord with the recent data of Claesson et al. [9] and of the European $4 \pi$ facility at SIS [28].

Note added in proof. We have learned that the $4 \pi \mathrm{Eu}-$ ropean Collaboration at GSI has very recently measured an excitation function of the baryonic entropy [29] over the (150-800) $\boldsymbol{A} \mathrm{MeV}$ energy range. Their results suggest low viscosity, possibly dependent on the impact energy.

\section{ACKNOWLEDGMENTS}

The authors wish to express their thanks to the members of the Plastic Ball and European $4 \pi$ collaborations for stimulating discussions. We also gratefully acknowledge original contributions made by G. Buchwald and G. Graebner. This work was supported by the Gesellschaft für Schwerionenforschung, the Bundesministerium für Forschung und Technologie, and by the Deutsche Forschungsgemeinschaft. 
[1] For reviews on nuclear fluid dynamics, see, e.g., J. A. Maruhn and W. Greiner, in Treatise on Heavy Ion Science, Vol. 4 of Extreme Nuclear States, edited by D. A. Bromley (Plenum, New York, 1985), p. 565; H. Stöcker and W. Greiner, Phys. Rep. 137, 277 (1986); R. B. Clare and D. Strottman, ibid. 141, 177 (1986); The Nuclear Equation of State, NATO ASI Series B: Physics Vol. 216A, edited by W. Greiner and H. Stöcker (Plenum, New York, 1989); J. A. Maruhn, in Relativistic Heavy Ion Physics, edited by L. P. Csernai and D. D. Strottman [Int. Rev. Nucl. Phys. 6 (1991)] (World Scientific, Singapore, 1991).

[2] W. Scheid and W. Greiner, Z. Phys. 226, 365 (1969); W. Scheid, H. Müller, and W. Greiner, Phys. Rev. Lett. 32, 741 (1974).

[3] H. G. Baumgardt, J. U. Schott, Y. Sakamoto, E. Schopper, H. Stöcker, J. Hofmann, W. Scheid, and W. Greiner, Z. Phys. A 237, 241 (1975).

[4] H. Stöcker, J. A. Maruhn, and W. Greiner, Z. Phys. A 293, 173 (1979); Phys. Rev. Lett. 44, 725 (1980).

[5] H. Stöcker, L. P. Csernai, G. Graebner, G. Buchwald, H. Kruse, R. Y. Cusson, J. A. Maruhn, and W. Greiner, Phys. Rev. C 25, 1873 (1982); G. Buchwald, G. Graebner, J. Theis, J. A. Maruhn, W. Greiner, H. Stöcker, K. Frankel, and M. Gyulassy, ibid. 28, 2349 (1983); G. Buchwald, G. Graebner, J. Theis, J. A. Maruhn, W. Greiner, and H. Stöcker, Phys. Rev. Lett. 52, 1594 (1984).

[6] H.-A. Gustafsson, H. H. Gutbrod, B. Kolb, H. Löhner, B. Ludewigt, A. M. Poskanzer, I. Renner, H. Riedesel, H. G. Ritter, A. Warwick, and H. Wieman, Phys. Rev. Lett. 52, 1590 (1984).

[7] K. G. R. Doss, H. A. Gustafsson, H. H. Gutbrod, K.-H. Kampert, B. Kolb, H. Löhner, B. Ludewigt, A. M. Poskanzer, H. G. Ritter, H. R. Schmidt, and H. Wieman, Phys. Rev. Lett. 57, 302 (1986); K. G. R. Doss, H. A. Gustafsson, H. H. Gutbrod, D. Hahn, K.-H. Kampert, B. Kolb, H. Löhner, A. M. Poskanzer, H. G. Ritter, H. R. Schmidt, and H. Stöcker, Phys. Rev. C 37, 163 (1988).

[8] D. L'Hôte, talk presented at the Fifth Gull Lake Nuclear Physics Conference, Gull Lake, Michigan, 1988 (unpublished); H. Gutbrod, K.-H. Kampert, B. Kolb, A. Poskanzer, H. Ritter, and H. R. Schmidt, Phys. Lett. B 216, 267 (1989); H. Gutbrod, K.-H. Kampert, B. Kolb, A. Poskanzer, H. Ritter, R. Schicker, and H. R. Schmidt, Phys. Rev. C 42, 640 (1990).

[9] G. Claesson, G. Krebs, J. Miller, G. Roche, L. S. Schroeder, W. Benenson, J. van der Plicht, J. S. Winfield, G. Landaud, J.-F. Gilot, C. Hartnack, and H. Stöcker, Phys. Lett. B 251, 23 (1990).

[10] H. H. Tang and C. Y. Wong, Phys. Rev. C 21, 1846 (1980).

[11] G. Buchwald, L. P. Csernai, J. A. Maruhn, W. Greiner, and H. Stöcker, Phys. Rev. C 24, 135 (1981).

[12] P. Danielewicz, Phys. Lett. 146B, 141 (1984).
[13] W. Schmidt, Ph.D. thesis, Johann Wolfgang GoetheUniversität, Frankfurt am Main, 1989 (unpublished).

[14] H. Kruse, B. V. Jacak, and H. Stöcker, Phys. Rev. Lett. 54, 289 (1985); J. Aichelin and G. Bertsch, Phys. Rev. C 31, 170 (1985); J. J. Molitoris and H. Stöcker, ibid. 32, 346 (1985); J. J. Molitoris, H. Stöcker, and B. L. Winer, ibid. 36, 220 (1987).

[15] J. Aichelin and H. Stöcker, Phys. Lett. 176B, 14 (1986); J. Aichelin, A. Rosenhauer, G. Peilert, H. Stöcker, and W. Greiner, Phys. Rev. Lett. 58, 1926 (1987).

[16] G. Peilert, H. Stöcker, W. Greiner, A. Rosenhauer, A. Bohnet, and J. Aichelin, Phys. Rev. C 39, 1402 (1989).

[17] L. P. Csernai, H. Stöcker, P. Subramanian, G. Buchwald, G. Graebner, A. Rosenhauer, J. A. Maruhn, and W. Greiner, Phys. Rev. C 28, 2001 (1983).

[18] H. Stöcker, J. Phys. G 10, L111 (1984).

[19] K.-H. Kampert, J. Phys. G 15, 691 (1989); H. Gutbrod, A. Poskanzer, and H. G. Ritter, Rep. Prog. Phys. 52, 1267 (1989).

[20] P. Siemens and J. Kapusta, Phys. Rev. Lett. 43, 1486 (1979); G. Bertsch and J. Cugnon, Phys. Rev. C 24, 2514 (1981); G. Bertsch, Nucl. Phys. A400, 221 (1983).

[21] H. Stöcker, G. Buchwald, G. Graebner, P. S. Subramanian, J. A. Maruhn, W. Greiner, B. Jacak, and G. D. Westfall, Nucl. Phys. A400, 63c (1983); D. Hahn and H. Stöcker, ibid. A476, 718 (1988); D. Hahn and H. Stöcker, Phys. Rev. C 37, 1048 (1988).

[22] P. Danielewicz and G. Odyniec, Phys. Lett. 157B, 146 (1985).

[23] K.-H. Kampert, Ph.D. thesis, Wilhelms-Universität zu Münster, 1986 (unpublished).

[24] B. Schürmann, Mod. Phys. Lett. A 3, 1137 (1988).

[25] P. Danielewicz and M. Gyulassy, Phys. Lett. 129B, 283 (1983).

[26] K. G. R. Doss, H. A. Gustafsson, H. H. Gutbrod, J. W. Harris, B. V. Jacak, K.-H. Kampert, B. Kolb, A. M. Poskanzer, H. G. Ritter, H. R. Schmidt, L. Teitelbaum, M. Tincknell, S. Weiss, and H. Wieman, Phys. Rev. Lett. 59, 2720 (1987).

[27] M. Berenguer, C. Hartnack, G. Peilert, H. Stöcker, W. Greiner, J. Aichelin, and A. Rosenhauer, J. Phys. G (in press); C. Hartnack, M. Berenguer, A. Jahns, A. v. Keitz, R. Mattiello, A. Rosenhauer, J. Schaffner, Th. Schönfeld, H. Sorge, L. Winckelmann, H. Stöcker, and W. Greiner, in Proceedings of the Fourth International Conference on Nucleus-Nucleus Collisions, Kanazawa, Japan, 1991, edited by H. Toki [Nucl. Phys. A (in press)].

[28] J. P. Coffin, Int. J. Mod. Phys. E1, 739 (1992); FOPI Collaboration, J. P. Alard et al., Phys. Rev. Lett. 69, 889 (1992).

[29] FOPI Collaboration, C. Kuhn et al., Phys. Rev. C (to be published). 